\title{
Comparison of gastrointestinal adverse effects between cyclooxygenase- 2 inhibitors and non-selective, non-steroidal anti-inflammatory drugs plus proton pump inhibitors
}

\author{
Shih-Wei Lai $\cdot$ Kuan-Fu Liao
}

Received: 11 January 2013/Accepted: 23 January 2013/Published online: 19 March 2013

(C) Springer Japan 2013

\section{Dear Sirs,}

We read with great interest a systematic review and meta-analysis recently published in J Gastroenterol [1], in which Jarupongprapa and coauthors reported that use of cyclooxygenase-2 inhibitors (COX-2 inhibitors) is significantly associated with decreased risk of major gastrointestinal events, including perforation, obstruction, and bleeding [relative risk $=0.38,95 \%$ confidence interval $(C I)=0.25-0.56]$. Similarly, Sakamoto and coauthors in Japan reported that use of celecoxib (a COX-2 inhibitor) for 2 weeks is less likely to develop gastroduodenal ulcers

An answer to this letter to the editor is available at doi:10.1007/s00535-013-0786-1.

\section{S.-W. Lai}

School of Medicine, China Medical University Hospital,

Taichung, Taiwan

e-mail: wei@www.cmuh.org.tw

\section{S.-W. Lai}

Department of Family Medicine,

China Medical University Hospital, Taichung, Taiwan

K.-F. Liao ( $\square)$

Graduate Institute of Integrated Medicine,

China Medical University, Taichung, Taiwan

e-mail: kuanfuliao@yahoo.com.tw

K.-F. Liao

Department of Internal Medicine, Taichung Tzu Chi General Hospital, No. 66, Sec. 1, Fongsing Road, Tanzi District,

Taichung City 427, Taiwan

K.-F. Liao

Department of Health Care Administration,

Central Taiwan University of Science and Technology,

Taichung, Taiwan than use of loxoprofen (a non-selective, non-steroidal antiinflammatory drug [ns-NSAID]) [3]. However, in a cohort study recently published in the Kuwait Medical Journal [2], we found that use of COX-2 inhibitors has a higher incidence of peptic ulcer disease than non-use of COX-2 inhibitors (19.28 vs. 5.91 per 1000 person-years, incidence rate ratio $=3.26,95 \%$ confidence interval $=2.63-4.04$ ). The subanalysis also demonstrated that use of celecoxib, meloxicam, nabumetone or nimesulide is significantly associated with increased risk of peptic ulcer disease, especially in those that are used for less than 3 months.

Based on the review by Jarupongprapa et al., COX-2 inhibitors seem to be superior to NSAIDs plus proton-pump inhibitors in mitigating the risk of major gastrointestinal events. Moreover, our study indicates that all COX-2 inhibitors are associated with increased risk of peptic ulcer disease. That is, COX-2 inhibitors are comparatively safe rather than undoubtedly safe. Therefore, prescription of any COX-2 inhibitor needs to be cautious in clinical practice.

Conflict of interest The authors disclose no conflicts of interest.

\section{References}

1. Jarupongprapa S, Ussavasodhi P, Katchamart W. Comparison of gastrointestinal adverse effects between cyclooxygenase-2 inhibitors and non-selective, non-steroidal anti-inflammatory drugs plus proton pump inhibitors: a systematic review and meta-analysis. J Gastroenterol. 2012. doi:10.1007/s00535-012-0717-6.

2. Lai SW, Liao KF, Lai HC, et al. Individual cyclooxygenase-2 inhibitors on the risk of peptic ulcer disease: a population-based cohort in Taiwan. Kuwait Med J. 2012;44:347-8.

3. Sakamoto C, Kawai T, Nakamura S, et al. Comparison of gastroduodenal ulcer incidence in healthy Japanese subjects taking celecoxib or loxoprofen evaluated by endoscopy: a placebocontrolled, double-blind 2-week study. Aliment Pharmacol Ther. 2013;37:346-54. 